\title{
ANÁLISE BIBLIOMÉTRICA DA PRODUÇÃO BRASILEIRA SOBRE A COVID-19
}

\section{BIBLIOMETRIC ANALYSIS OF BRAZILIAN \\ PRODUCTION ON COVID-19}

\section{ANÁLISIS BIBLIOMÉTRICO DE LA PRODUCCIÓN BRASILEÑA EN COVID-19}

\author{
Wilton Nascimento Figueredo ${ }^{1}$ \\ Tássia Teles Santana de Macêdo ${ }^{2}$ \\ Gustavo Marques Porto Cardoso ${ }^{3}$ \\ Elionara Teixeira Boa Sorte Fernandes ${ }^{4}$
}

\begin{abstract}
Como citar esse artigo: Figueredo WN, Macêdo TTS, Cardoso GMP, Fernandes ETBS. Análise bibliométrica da produção brasileira sobre a COVID-19. Rev baiana enferm. 2020;34:e37107.

Objetivo: analisar a produção científica brasileira sobre a COVID-19. Método: pesquisa bibliográfica, descritiva, quantitativa e de análise bibliométrica. A fonte de informação foi a PubMed, com recorte temporal entre 17 de novembro 2019 e 18 de maio 2020. Foram utilizados para a análise dos dados os softwares RSudio e VOSviewer, os pacotes bibliométricos bibliometrix e biblioshiny, e o site Word Cloud Art. Resultados: os autores brasileiros identificados nos 248 artigos são, em sua maioria, da área da medicina, atuando em instituições nas Regiões Sudeste e Nordeste do Brasil e mantêm colaboração internacional, principalmente entre os Estados Unidos, Itália e Canadá. Há publicação em diversos periódicos, com destaque para os de origem brasileira. As palavras-chave e os artigos publicados remetem às temáticas biomédicas. Conclusão: a produção científica brasileira carece de pesquisas sobre a COVID-19 que reflitam sobre os impactos da pandemia para a sociedade, os trabalhadores e a economia da saúde no Brasil.
\end{abstract}

Descritores: COVID-19. SARS-CoV-2. Betacoronavírus. Bibliometria. Brasil.

Objective: to analyze the Brazilian scientific production on COVID-19. Method: bibliographic, descriptive, quantitative and bibliometric analysis research. The source of information was PubMed, with a period between November 17, 2019 and May 18, 2020. RSudio and VOSviewer software, bibliometrix and biblioshiny bibliometric packages, and Word Cloud Art were used for data analysis. Results: the Brazilian authors identified in the 248 articles are mostly from the medical area, working in institutions in the Southeast and Northeast regions of Brazil and maintain international collaboration, mainly with the United States, Italy and Canada. There is publication in several journals, especially those of Brazilian origin. The keywords and published articles refer to biomedical themes. Conclusion: Brazilian scientific production lacks researches on COVID-19 that reflect on the impacts of the pandemic on society, workers and health economy in Brazil.

Descriptors: COVID-19. SARS-CoV-2. Betacoronavirus. Bibliometrics. Brazil.

\footnotetext{
Enfermeiro. Mestre em Estudos Interdisciplinares sobre a Universidade. Doutor em Enfermagem. Professor da Universidade Estadual de Feira de Santana. Feira de Santana, Bahia, Brasil.wnfigueredo@uefs.br.http://orcid.org/0000-0003-2066-09|4.

Enfermeira. Doutora e Mestre em Enfermagem. Professora Assistente na Escola Bahiana de Medicina e Saúde Pública. Salvador, Bahia, Brasil. http://orcid.org/00000003-2423-9844.

3 Educação Física. Mestre em Estudos Interdisciplinares sobre a Universidade. Professor Assistente de Educação Física da Faculdade Nobre de Feira de Santana. Feira de Santana, Bahia, Brasil. http://orcid.org/0000-0002-0I25-6492.

4 Enfermeira. Doutora e Mestre em Enfermagem. Professora Assistente na Universidade do Estado da Bahia, Campus XII. Guanambi, Bahia, Brasil. http://orcid. org/0000-000 I-8302-6887.
} 
Objetivo: analizar la producción científica brasileña en COVID-19. Método: investigación bibliográfica, descriptiva, cuantitativa y de análisis bibliométrico. La fuente de información fue PubMed, con un periodo entre el 17 de noviembre de 2019 y el 18 de mayo de 2020. Para el análisis de datos, se utilizaron el software RSudio y VOSviewer, los paquetes bibliométricos bibliometrix y biblioshiny, y Word Cloud Art. Resultados: los autores brasileños identificados en los 248 artículos son en su mayoría del área de la medicina, trabajando en instituciones en las regiones sureste y noreste de Brasil y mantienen la colaboración internacional, principalmente entre los Estados Unidos, Italia y Canadá. Hay publicación en varias revistas, especialmente las de origen brasileño. Las palabras clave y los artículos publicados se refieren a temas biomédicos. Conclusión: la producción científica brasileña carece de investigaciones sobre COVID-19 que reflexionen sobre los impactos de la pandemia en la sociedad, los trabajadores y la economía de la salud en Brasil.

Descriptores: COVID-19. SARS-CoV-2. Betacoronavirus. Bibliometría. Brasil.

\section{Introdução}

No Brasil, desde a primeira notificação em 26 de fevereiro de 2020 até primeiro de junho do mesmo ano, um total de 526.447 casos da COVID-19 foram confirmados, e desses, 29.937 foram óbitos ${ }^{(1)}$. Segundo o consolidado da distribuição da COVID-19 pelo mundo e atualizado diariamente pela European Centre for Disease Prevention and Control (ECDC) ${ }^{(2)}$, o Brasil é o segundo e o quarto país a liderar a estatística mundial das infecções notificadas e óbitos confirmados, respectivamente.

A atual pandemia, causada pela síndrome respiratória coronavírus $2^{(3)}$ (SARS-CoV-2) ou COVID-19 e declarada pela Organização Mundial de Saúde (OMS) em março de 2020, é responsável pela infecção de quase cinco milhões de pessoas em todo o mundo ${ }^{(2)}$. O vírus SARS-CoV-2 possui alta taxa de infectividade e, por isso, o sistema de saúde de vários países ${ }^{(4-5)}$ tem entrado em estado de emergência devido às inúmeras internações hospitalares. Inclusive com um número elevado de contaminação por profissionais da saúde, principalmente o pessoal da área da enfermagem brasileira, com mais de cinco mil casos confirmados ${ }^{(6)}$.

Diante desse cenário, a comunidade científica de diversas áreas, como por exemplo, epidemiologia $^{(7)}$, medicina ${ }^{(8)}$, saúde pública $^{(9)}$ e economia ${ }^{(10)}$, está empenhada em pesquisar, publicar e consolidar evidências científicas, que são imprescindiveis para a orientação da população, dos profissionais e dos gestores de saúde, objetivando assim, a construção de estratégias seguras e eficazes para o enfrentamento da pandemia.
Nessa perspectiva, conhecer a tendência das publicações científicas, a identificação das lacunas, é essencial para a comunicação científica, bem como para a averiguação da qualidade das pesquisas. Assim, a análise bibliométrica se constitui como ferramenta indispensável para a avaliação dessas publicações.

A análise bibliométrica é uma técnica útil para aferir quantitativamente e objetivamente a pesquisa atual de um determinado assunto e sua influência local ou mundial com aspecto da qualidade científica ${ }^{(11-12)}$. Os indicadores criados pela bibliometria têm a finalidade de mostrar resultados imediatos para subsidiar a ciência e a tecnologia, e a consequente criação de políticas públicas ${ }^{(11-12)}$. No entanto, até onde se sabe, foram localizados três artigos ${ }^{(13-15)}$ da produção mundial e não há evidências anteriores de uma análise bibliométrica local brasileira sobre a temática da COVID-19.

Tendo em vista essas considerações, a pergunta investigativa que norteou a produção deste artigo foi: O que está sendo produzido pelos pesquisadores brasileiros sobre a temática da COVID-19?

Com o intuito de responder a pergunta investigativa, este estudo objetiva analisar a produção científica brasileira sobre a COVID-19.

\section{Método}

Pesquisa bibliográfica, descritiva, com abordagem quantitativa e que utilizou o método de 
análise bibliométrica. A bibliometria contribui estatisticamente para avaliar tendências de crescimento da produção, publicação, autoria e uso sobre determinada temática pesquisada em diversas áreas do saber ${ }^{(16)}$.

Com base em outras pesquisas ${ }^{(17-18)}$, este estudo seguiu cinco etapas: objetivo da pesquisa, protocolo da pesquisa, coleta dos dados, análise dos dados e resumo dos resultados.

A definição do tema, o objetivo e a questão norteadora da pesquisa, apresentados na introdução, compuseram a primeira etapa deste estudo bibliométrico.

$\mathrm{Na}$ segunda etapa, denominada de protocolo de pesquisa, foram definidas a base de dados, as palavras-chave e a estratégia de busca. A PubMed, base de dados da National Center for Biotechnology Information (NCBI), foi escolhida por abranger os documentos da Medical Literature Analysis and Retrieval System Online (MEDLINE) e compreender mais de 30 milhões de citações para a literatura biomédica, enfermagem, farmacologia, odontologia, veterinária e outras áreas de saúde. As palavras-chave no idioma inglês: COVID-19, SARS-CoV-2, Coronavirus e Brazil foram definidas intencionadas ao objeto de estudo e fazendo o cruzamento com os caracteres booleanos $O R$ e $A N D$. A estratégia de busca utilizada foi: "COVID-19" $O R$ "SARS-CoV-2" OR "Coronavirus" AND "Brazil".

$\mathrm{Na}$ terceira etapa, coleta dos dados, foram incluídos todos os documentos indexados no período entre 17 de novembro de 2019 e 18 de maio de 2020, o que compreende 6 meses desde os primeiros rumores sobre a COVID-19 até a data de coleta na base de dados. Não houve exclusão de documentos, pois a PubMed indexa apenas artigos originais ou de revisão. Ainda nessa etapa, foi construído o banco de dados por meio do programa Excel ${ }^{\circledR}$ (versão 2016). As informações do Journal Impactor Factor (JIF) e índice-H dos autores foram extraídas da base Journal Citation Reports da Clarivate Analytics e da base de dados Scopus, respectivamente. A área de formação dos autores foi coletada na plataforma do currículo lattes.

Durante a quarta etapa, análise dos dados, foram utilizados os softwares RStudio ${ }^{\circledR}$ (versão
3.6.1) e VOSviewer ${ }^{\circledR}$ (versão 1.6.6). Para a execução da análise bibliométrica, em interface ao RStudio, foram instalados os pacotes bibliometrix e biblioshiny. Com os pacotes e metadados instalados, foi possível acessar o número total de publicações/ano, nome do autor, países/regiões, métricas de autoria, fontes de periódicos e palavras-chave. Por meio do VOSviewer foi analisada a rede de colaboração por coautoria e por coocorrência das palavras-chave. A frequência das palavras nos resumos dos artigos foi apresentada graficamente pela plataforma on-line Word Cloud Art.

Para a disposição e apresentação dos resultados, última etapa do método bibliométrico, optou-se por utilizar as figuras geradas pelos pacotes citados e as tabelas criadas pelo Excel. Devido ao grande número de documentos, escolheu-se apresentar os dez termos (ranking Top 10) com maior prevalência. Além disso, para a classificação do Top 10 dos autores mais produtivos foi considerado o maior número de artigos e o índice-H, nessa ordem. Para a identificação da afiliação dos autores foi levado em consideração a primeira instituição relatada no artigo.

Os elementos analisados neste artigo são: distribuição de autoria, colaboração internacional entre os autores, periódicos de maior relevância e JIF, coocorrência das palavras-chave e artigos mais citados com a frequência das palavras em seus resumos.

Por se tratar de um estudo que utiliza dados secundários e disponíveis gratuitamente em repositório de dados, não houve necessidade de submissão ao Comitê de Ética em Pesquisas (CEP).

\section{Resultados}

Foram identificados 248 artigos com a participação de autores brasileiros, publicados em 155 periódicos, coautoria de 1.585 pesquisadores e afiliados em diversas instituições distribuídas em 26 países.

Os autores localizados em instituições das Regiões Sudeste e Nordeste concentram 90\% das publicações, do total de 10 autores analisados, conforme Tabela 1. A média de publicação e do índice-H dos autores foi de aproximadamente 5 e 17, respectivamente. A Medicina correspondeu 
a 60\% da área de formação dos autores. Destaca-se ainda que, entre os autores mais produtivos, 30\% não possuíam nacionalidade brasileira, estando vinculados à respectiva instituição brasileira por contrato como professor visitante ou pós-doutorado.

Tabela 1 - Top 10 dos autores mais produtivos sobre a COVID-19 em publicações brasileiras, instituição/afiliação, região, índice-H, nacionalidade e área de formação. Salvador, Bahia, Brasil - 2020

\begin{tabular}{|c|c|c|c|c|c|c|}
\hline Autores & Instituição/Afiliação & Região & $\mathbf{N}$ & Índice-H & Nacionalidade & $\begin{array}{c}\text { Área de } \\
\text { formação }\end{array}$ \\
\hline Giovanetti M & Fundação Oswaldo Cruz & Sudeste & 9 & 15 & Italiana & Biologia \\
\hline Rolim-Neto ML & $\begin{array}{l}\text { Escola de Medicina } \\
\text { Juazeiro do Norte }\end{array}$ & Nordeste & 8 & 4 & Brasileira & Psicologia \\
\hline Hussain A & $\begin{array}{l}\text { Universidade Federal do } \\
\text { Ceará }\end{array}$ & Nordeste & 5 & 25 & Norueguesa & Medicina \\
\hline Kroumpouzos G & $\begin{array}{l}\text { Escola de Medicina } \\
\text { Jundiaí }\end{array}$ & Sudeste & 5 & 15 & Norte-Americana & Medicina \\
\hline Castro R & Fundação Oswaldo Cruz & Sudeste & 5 & 7 & Brasileira & Odontologia \\
\hline Da Silva CGL & $\begin{array}{l}\text { Escola de Medicina } \\
\text { Juazeiro do Norte }\end{array}$ & Nordeste & 5 & 5 & Brasileira & Medicina \\
\hline Kowalski LP & $\begin{array}{l}\text { Universidade de São } \\
\text { Paulo }\end{array}$ & Sudeste & 4 & 63 & Brasileira & Medicina \\
\hline Croda J & $\begin{array}{l}\text { Universidade Federal do } \\
\text { Mato Grosso do Sul }\end{array}$ & $\begin{array}{l}\text { Centro- } \\
\text { Oeste }\end{array}$ & 4 & 18 & Brasileira & Medicina \\
\hline Perazzo H & Fundação Oswaldo Cruz & Sudeste & 4 & 14 & Brasileira & Medicina \\
\hline Martins-Filho PR & $\begin{array}{l}\text { Universidade Federal do } \\
\text { Sergipe }\end{array}$ & Nordeste & 4 & 13 & Brasileira & Odontologia \\
\hline
\end{tabular}

Fonte: Elaboração própria.

A colaboração internacional dos autores brasileiros foi evidenciada principalmente entre os países dos Estados Unidos (51 artigos), Itália (44 artigos) e Canadá (24 artigos). Na Figura 1, é possível verificar o fluxo da distribuição geográfica mundial dessa colaboração.

Figura 1 - Distribuição geográfica da colaboração internacional entre os autores

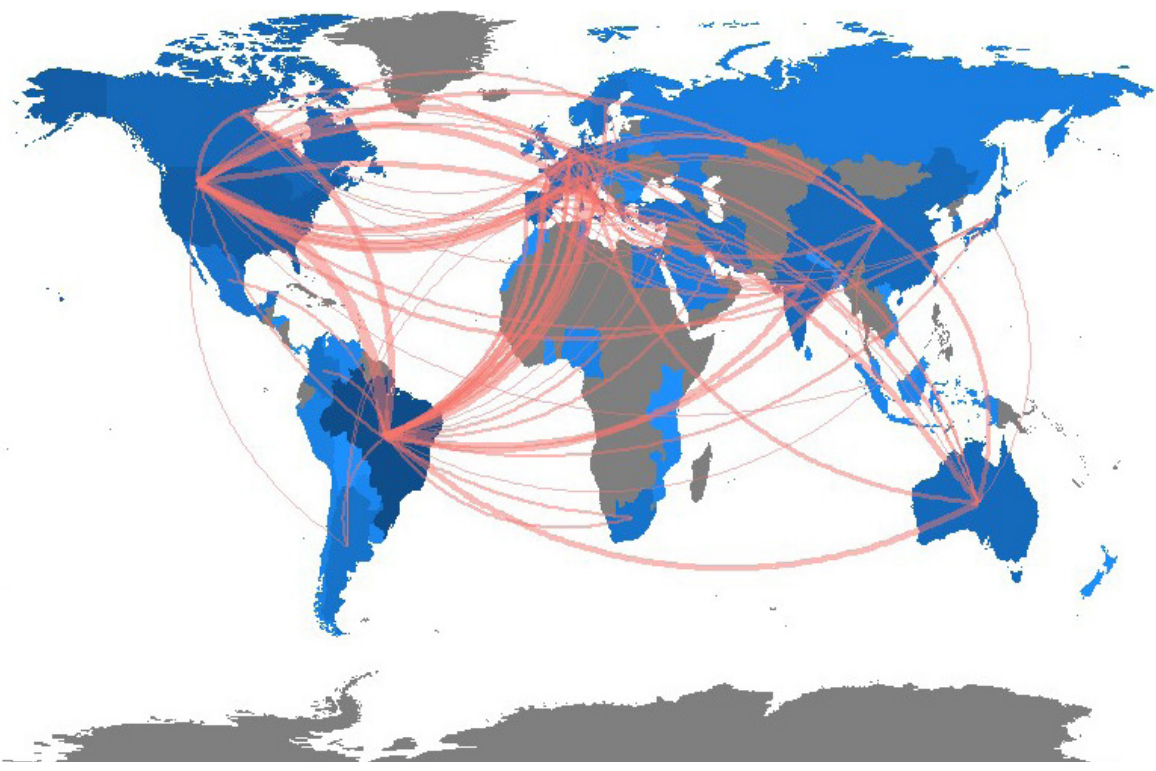

Fonte: Elaboração própria. 
Conforme se verifica na Tabela 2, o periódico Cadernos de Saúde Pública concentra as maiores publicações de autores brasileiros sobre a temática da COVID-19. Entretanto, em termos do fator cientométrico de citação, no JIF - que reflete o número médio de citações que os artigos receberam nesse periódico - o periódico Cadernos de Saúde Pública ocupa o último lugar em relação às outras revistas descritas.

Tabela 2 - Top 10 dos periódicos com maior número de artigos publicados sobre COVID-19 de acordo com o Journal Impactor Factor. Salvador, Bahia, Brasil - 2020

\begin{tabular}{l|cc}
\hline Revistas & N & JIF \\
\hline Cadernos de Saúde Pública & 9 & 1.170 \\
Psychiatry Research & 9 & 2.208 \\
Journal of Medical Virology & 8 & 2.049 \\
Science of The Total Environment & 8 & 5.589 \\
Travel Medicine and Infectious Disease & 6 & 4.868 \\
Dermatologic Therapy & 5 & 1.740 \\
Oral Oncology & 5 & 3.730 \\
Epidemiologia e Serviços de Saúde: Revista do SUS Brasil & 4 & NE \\
Revista da Sociedade Brasileira de Medicina Tropical & 4 & 1.498 \\
Data in Brief & 3 & NE \\
\hline
\end{tabular}

Fonte: Elaboração própria.

Legenda: JIF: Journal Impactor Factor - 2018; NE: Não encontrado.

Na Figura 2 é possível identificar a formação das palavras-chave por meio de nós que se conectam em redes de três cores: verde, vermelho e azul. O tamanho do nó e do item indicam a frequência de ocorrência de uma palavra-chave e sua relação é mais forte quanto mais próximos entre eles. O conjunto verde, possui sete palavras-chave e abrange o termo COVID-19, seus sinônimos, epidemiologia e recomendação global para o enfrentamento da pandemia, como a quarentena. O conjunto da cor vermelha refere-se aos descritores relacionados ao subtipo do coronavírus e as infecções respiratórias secundárias ao vírus. Enquanto o conjunto da cor azul inclui os termos relacionados às características demográficas e a localidade dos estudos sobre a COVID-19.

Figura 2 - Coocorrência das palavras-chave entres os artigos

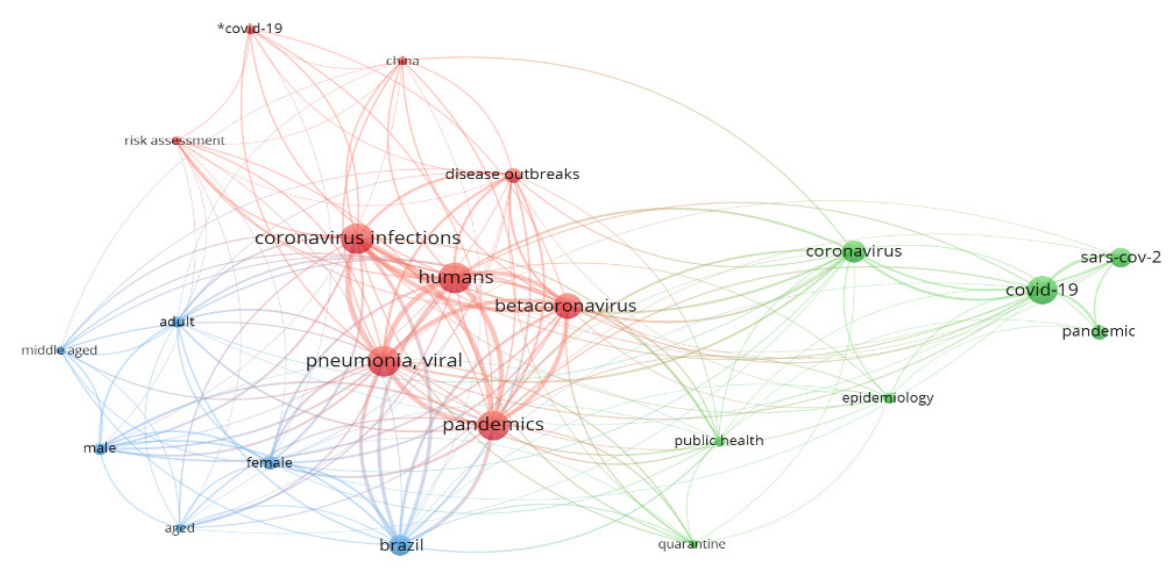

\& Vosviewer

Fonte: Elaboração própria. 
Entre os dez $\operatorname{artigos}^{(19-28)}$ mais citados, percebe-se a hegemonia da área da Biologia e da coautoria de Giovanetti, de nacionalidade italiana, em 60\% nas publicações. O resultado da frequência das palavras na Figura 3 denota que a principal discussão temática está direcionada para a codificação, sequenciamento do genoma do SARS-CoV-2. Além disso, também se evidencia palavras que denotam as recomendações para a prevenção durante a pandemia, como medidas de isolamento e do contato humano.

Figura 3 - Frequência das palavras mais citadas nos resumos dos artigos com maiores citações

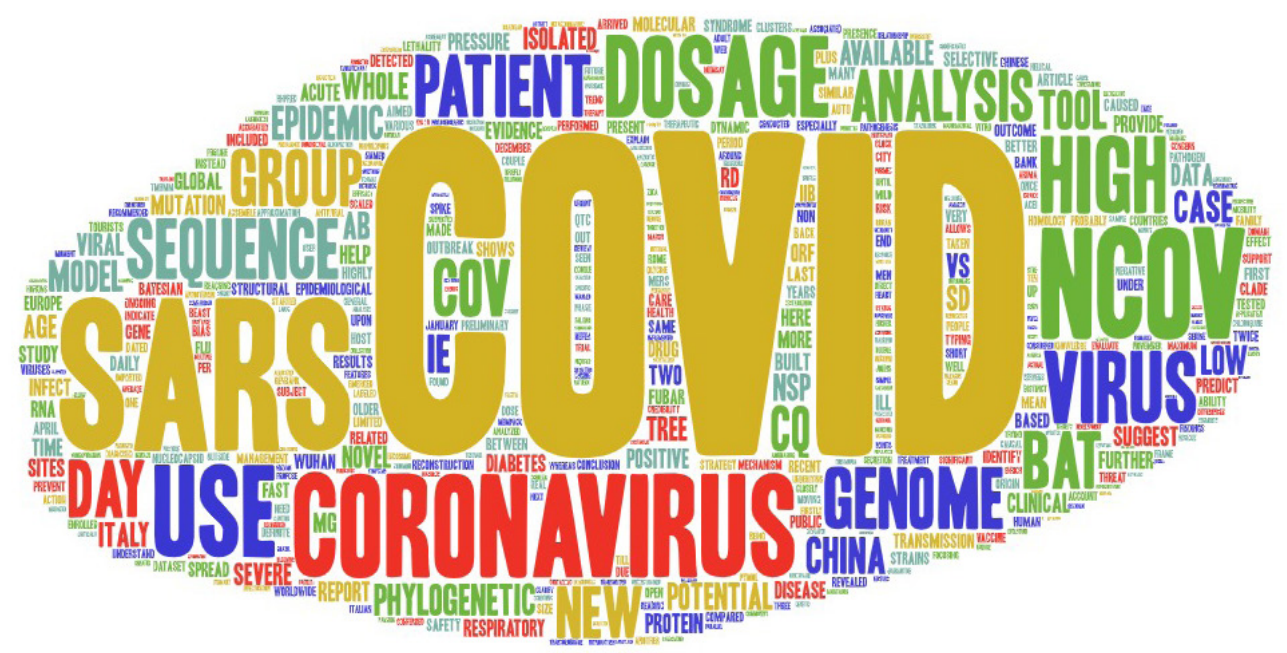

Fonte: Elaboração própria.

\section{Discussão}

A comunidade científica brasileira tem acompanhado a magnitude da importância de discussões direcionadas ao enfrentamento da pandemia causada pela COVID-19. Entretanto, em relação ao volume da produção mundial ${ }^{(13,15)}$, o Brasil não tem contado com significativas publicações. Acredita-se que o contingenciamento das verbas nas universidades ${ }^{(29)}$, a falta de financiamento para pesquisadores cientistas brasileiros $^{(30)}$, a passividade e o obscurantismo da atual política ${ }^{(9,31)}$ possam ter impacto direto para o desenvolvimento científico de pesquisas brasileiras sobre SARS-CoV-2.

O desenvolvimento científico e tecnológico brasileiro, majoritariamente, sempre esteve presente nas Regiões Sudeste e Sul ${ }^{(32)}$, com essas regiões liderando as estatísticas de pesquisas e a produção científica. Entretanto, neste estudo, os pesquisadores localizados em instituições da Região Nordeste ultrapassaram as publicações sobre a COVID-19 da Região Sul. A liderança da
Região Nordeste deve-se ao incentivo das instituições de ensino em propiciar a abertura de novas vagas na pós-graduação, ao incremento de parcerias interinstitucionais ${ }^{(32)}$ e à internacionalização de suas ações.

A necessidade da internacionalização da educação de ensino superior é fator crucial para o desenvolvimento de pesquisas. A internacionalização ou modalidade ativa recepção de pesquisadores estrangeiros no Brasil - se constitui como forte indicador e propulsor para o desenvolvimento científico, tecnológico e cultural ${ }^{(33)}$. Assim como a modalidade ativa, a internacionalização passiva envio de pesquisadores brasileiros para o exterior - faz-se importante para criar, ampliar e consolidar as redes de cooperação entre as instituições. Neste estudo, a internacionalização, principalmente a modalidade passiva, com a colaboração internacional entre pesquisadores italianos, contribuiu para que o Brasil entrasse no ranking dos artigos publicados e de citações sobre a temática da COVID-19. 
Contudo, neste estudo, ainda se verificam assimetrias entre as cinco regiões brasileiras, entre os pesquisadores e as áreas de maior domínio da produção científica. As publicações científicas continuam em um grupo seletivo de pesquisadores com alta produtividade na área da Medicina, como se verificou no índice-H. Apesar do índice-H ser controverso ${ }^{(34)}$ entre a comunidade científica, ele quantifica pelas citações das produções de um determinado pesquisador, mas não mede os valores qualitativos da ciência, como ocorre na área da enfermagem ${ }^{(35)}$. A enfermagem é tão essencial quanto a medicina nesse momento de pandemia, principalmente pelo seu protagonismo no cuidado aos pacientes sintomáticos e hospitalizados pela COVID-19. Nessa perspectiva, faz-se necessário focar pesquisas direcionadas a outras vertentes da SARS-CoV-2, como, por exemplo, o impacto da pandemia no cotidiano dos profissionais da enfermagem e a sistematização do cuidado, já que não foi verificada nenhuma publicação no Top 10 com autores da respectiva área.

Investir em pesquisa é ampliar as possibilidades de produção científica, além de orientar os tomadores de decisão para o planejamento de ações, sistemas e serviços de saúde. No cenário atual, os países que mais publicaram sobre a COVID-19 estão situados na América do Norte, Europa e Ásia. Esses países lideram a corrida tecnológica e priorizam investimentos em pesquisa e, por consequência, lideram o ranking de publicações ${ }^{(14)}$.

A pandemia do coronavírus gerou um aumento no volume de publicações ${ }^{(13)}$ que utilizam principalmente o termo COVID-19 como o seu principal descritor. Pela análise das Figura 2 e 3, é possível verificar a valorização de estudos sobre uma tendência biomédica, com terminologias como vírus, doença e tratamento. Não obstante ser extremamente importante a cura ou uma vacina para a doença, é necessário pesquisar sobre os impactos que a pandemia causará na saúde mental das pessoas ${ }^{(22)}$, no setor da economia e educação ${ }^{(10)}$, por exemplo.

Em outra perspectiva, entre os periódicos classificadas no Top 10 (Tabela 2), verifica-se o interesse dos pesquisadores brasileiros em revistas com escopo dos mais variados, com questões voltadas para a saúde pública e pesquisas clínicas. Entretanto, o periódico com maior número de publicações sobre a COVID-19, de origem brasileira, é o que possui menor classificação no JIF. Esse fato denota que, talvez, o idioma utilizado pela revista interfira no acesso, na leitura e na citação por outros pesquisadores internacionais.

As limitações deste estudo são decorrentes da escolha de uma única base de dados, das palavras-chave e do uso dos pacotes bibliométricos. A escolha da base de dados e das palavras-chave podem ter camuflado estudos com a mesma temática e não indexados na mesma base. Os pacotes bibliométricos utilizados são limitados quanto à aplicação de filtros e análises mais robustas. Além disso, a apresentação do ranking Top 10 pode ser uma limitação, já que apenas os dez primeiros itens foram considerados. Assim, sugere-se outras investigações semelhantes, pós período pandêmico, cruzando outras bases de dados, para se investigar a produção brasileira sobre a COVID-19.

Por meio do panorama dos últimos seis meses da produção científica brasileira sobre a COVID-19, este estudo traz como contribuições a apresentação do foco das pesquisas e as lacunas de conhecimento sobre a temática SARS-CoV-2. Além disso, destaca-se a importância de colaborações internacionais para o crescimento da produção brasileira em âmbito mundial.

\section{Conclusão}

Conclui-se que a produção científica brasileira sobre a COVID-19 tem como área hegemônica a medicina, com temáticas essencialmente biomédicas, direcionados para o sequenciamento do genoma do vírus SARS-CoV-2, da cura da doença e para estudos observacionais clínicos. Ao mesmo tempo, a produção científica dos pesquisadores brasileiros é mantida por meio de colaboração internacional entre diversos países e carecem pesquisas sobre a COVID-19 que 
reflitam sobre os impactos da pandemia para a sociedade, para os trabalhadores e a economia da saúde no Brasil.

Entretanto, a análise bibliométrica evidenciou que é preciso estimular novas pesquisas e produção científica em outras áreas sobre a temática da COVID-19, como, por exemplo, a área da enfermagem, no campo da saúde mental e dos impactos sociais e econômicos para a área da saúde no Brasil.

\section{Colaborações:}

1 - Concepção, projeto, análise e interpretação dos dados: Wilton Nascimento Figueredo e Tássia Teles Santana de Macêdo;

2 - Redação do artigo e revisão crítica relevante do conteúdo intelectual: Wilton Nascimento Figueredo, Tássia Teles Santana de Macêdo, Gustavo Marques Porto Cardoso e Elionara Teixeira Boa Sorte Fernandes;

3 - Aprovação final da versão a ser publicada: Wilton Nascimento Figueredo, Tássia Teles Santana de Macêdo, Gustavo Marques Porto Cardoso e Elionara Teixeira Boa Sorte Fernandes.

\section{Referências}

1. Ministério da Saúde. Painel Coronavírus [Internet]. Brasília (DF); 2020 [cited 2020 May 20]. Available from: https://covid.saude.gov.br/

2. European Centre for Disease Prevention and Control. Download today's data on the geographic distribution of COVID-19 cases worldwide [Internet]. Estocolmo (SWE); 2020 [cited 2020 May 20]. Available from: https://www.ecdc.europa. eu/en/publications-data/download-todays-datageographic-distribution-covid-19-cases-worldwide

3. Gorbalenya AE, Baker SC, Baric RS, Groot RJ, Drosten C, Gulyaeva AA, et al. The species Severe acute respiratory syndrome-related coronavirus: classifying 2019-nCoV and naming it SARS-CoV-2. Nat Microbiol. 2020;5(4):536-44. DOI: 10.1038/ s41564-020-0695-Z\%0A

4. Spina S, Marrazzo F, Migliari M, Stucchi R, Sforza A, Fumagalli R. The response of Milan's
Emergency Medical System to the COVID-19 outbreak in Italy. Lancet. 2020;395(10227):e49-50. DOI:10.1016/S0140-6736(20)30493-1

5. Zhang S, Wang Z, Chang R, Wang H, Xu C, Yu X, et al. COVID-19 containment: China provides important lessons for global response. Front Med. 2020;14(2):215-9. DOI: 10.1007/s11684-020-0766-9

6. Conselho Federal de Enfermagem. Observatório da Enfermagem [Internet]. Brasilia (DF); 2020 [cited 2020 Jun 1]. Available from: http:// observatoriodaenfermagem.cofen.gov.br

7. Xu B, Gutierrez B, Mekaru S, Sewalk K, Goodwin L, Loskill A, et al. Epidemiological data from the COVID-19 outbreak, real-time case information. Sci Data. 2020;7(1):1-6. DOI: 10.1038/ s41597-020-0448-0

8. Zhang HW, Yu J, Xu HJ, Lei Y, Pu ZH, Dai WC, et al. Corona Virus International Public Health Emergencies: Implications for Radiology Management. Acad Radiol. 2020;27(4):463-7. DOI: 10.1016/j.acra.2020.02.003

9. Campos GWS. O pesadelo macabro da Covid-19 no Brasil: entre negacionismos e desvarios. Trab educ saúde. 2020;18(3):1-5. DOI: 10.1590/1981-7746-sol00279

10. McKibbin WJ, Fernando R. The Global Macroeconomic Impacts of COVID-19: Seven Scenarios. CAMA Work Pap. 2020;19:1-45. DOI: $10.2139 /$ ssrn. 3547729

11. Avena MJ, Barbosa DA. Bibliometric indicators of the nursing journals according to the index databases. Rev Esc Enferm USP. 2017;51:e03262. DOI: $10.1590 / \mathrm{s} 1980-220 x 2017014603262$

12. Giménez-Espert M del C, Prado-Gascó VJ. Bibliometric analysis of six nursing journals from the Web of Science, 2012-2017. J Adv Nurs. 2019;75(3):543-54. DOI: 10.1111/jan.13868

13. Chahrour M, Assi S, Bejjani M, Nasrallah AA, Salhab H, Fares MY, et al. A Bibliometric Analysis of COVID-19 Research Activity: A Call for Increased Output. Cureus. 2020;12(3):1-8. DOI: 10.7759/cureus. 7357

14. De Melo MC, Cabral ERM, Rolim ACA, Oliveira REM, Takahashi F, Araujo AC, et al. Uma análise bibliométrica das pesquisas globais da COVID-19. Int J Med Heal. 2020;3:e202003019. DOI: $10.31005 /$ jmh.v3i0.88 
Wilton Nascimento Figueredo, Tássia Teles Santana de Macêdo, Gustavo Marques Porto Cardoso, Elionara Teixeira Boa Sorte Fernandes

15. O`Brien N, Barboza-Palomino M, Ventura-León J, Caycho-Rodríguez T, Sandoval-Díaz JS, López-López W, et al. Nuevo coronavirus (COVID-19). Un análisis bibliométrico. Rev Chil Anest. 2020;49(3):408-15. DOI: 10.25237/ revchilanestv $49 \mathrm{n} 03.020$

16. Fonseca EN. Bibliometria: teoria e prática. São Paulo: Cultrix; 1986.

17. Troian A, Gomes MC. A bibliometric analysis on the use of the multicriteria approach to the water resource management. Gestão \& Produção. 2020;27(2):1-18. DOI: 10.1590/0104-530x4761-20

18. Wingerter DG, Azevedo UN, Marcacini AM, Alves M do SCF, Ferreira MÂF, Moura LKB. Produção científica sobre quedas e óbitos em idosos: Uma análise bibliométrica. Rev Bras Geriatr Gerontol. 2018;21(3):331-40. DOI: 10.1590/1981-22562018021.170168

19. Benvenuto D, Giovanetti M, Ciccozzi A, Spoto S, Angeletti S, Ciccozzi M. The 2019-new coronavirus epidemic: Evidence for virus evolution. J Med Virol. 2020;92(4):455-9. DOI: 10.1002/jmv.25688

20. Giovanetti M, Benvenuto D, Angeletti S, Ciccozzi M. The first two cases of 2019-nCoV in Italy: Where they come from? J Med Virol. 2020;92(5):518-21. DOI: 10.1002/jmv.25699

21. Angeletti S, Benvenuto D, Bianchi M, Giovanetti M, Pascarella S, Ciccozzi M. COVID2019: The role of the nsp2 and nsp3 in its pathogenesis. J Med Virol. 2020;92(6):584-8. DOI: 10.1002/jmv.25719

22. Lima CKT, Carvalho PMM, Lima IAAS, Nunes JVAO, Saraiva JS, de Souza RI, et al. The emotional impact of Coronavirus 2019-nCoV (new Coronavirus disease). Psychiatry Res. 2020;287:112915. DOI: 10.1016/j. psychres.2020.112915

23. Benvenuto D, Giovanetti M, Vassallo L, Angeletti S, Ciccozzi M. Application of the ARIMA model on the COVID-2019 epidemic dataset. Data Br. 2020;29:105340. DOI: 10.1016/j. dib.2020.105340

24. Borba MGS, Val FFA, Sampaio VS, Alexandre MAA, Melo GC, Brito $\mathrm{M}$, et al. Effect of High vs Low Doses of Chloroquine Diphosphate as Adjunctive Therapy for Patients Hospitalized With Severe Acute Respiratory Syndrome Coronavirus 2 (SARS-CoV-2)
Infection: A Randomized Clinical Trial. JAMA Netw Open. 2020;3(4):e208857. DOI: 10.1001/ jamanetworkopen.2020.8857

25. Benvenuto D, Giovanetti M, Salemi M, Prosperi M, De Flora C, Junior Alcantara LC, et al. The global spread of 2019-nCoV: a molecular evolutionary analysis. Pathog Glob Health. 2020;114(2):64-7. DOI: $10.1080 / 20477724.2020 .1725339$

26. Hussain A, Bhowmik B, Moreira NC do V. COVID-19 and Diabetes: Knowledge in Progress. Diabetes Res Clin Pract. 2020. DOI: 10.1016/j. diabres.2020.108142

27. Cleemput S, Dumon W, Fonseca V, Karim WA, Giovanetti M, Alcantara LC, et al. Genome Detective Coronavirus Typing Tool for rapid identification and characterization of novel coronavirus genomes. Bioinformatics. 2020:1-4. DOI: 10.1093/bioinformatics/btaa145

28. Giovanetti M, Angeletti S, Benvenuto D, Ciccozzi M. A doubt of multiple introduction of SARS-CoV-2 in Italy: A preliminary overview. J Med Virol. 2020: 1-3. DOI: 10.1002/jmv.25773

29. Buarque C. Outros contingenciamentos. Rev Práticas Adm Pública [Internet]. 2019 [cited 2020 May 20];3(3):33-40. Available from: https:// periodicos.ufsm.br/pap/article/view/43375/23917

30. Sociedade Brasileira para o Progresso da Ciência. Klebis D, editor. Jornal da Ciência [Internet]. 2019 [cited 2020 May 20]; (785):1-20. Available from: http://sbpcacervodigital.org.br/ handle/20.500.11832/4977

31. Burki T. COVID-19 in Latin America. Lancet Infect Dis. 2020;20(5):547-8. DOI: 10.1016/ S1473-3099(20)30303-0

32. Sidone OJG, Haddad EA, Mena-Chalco JP. A ciência nas regiões brasileiras: evolução da produção e das redes de colaboração científica. TransInformação. 2016;28(1):15-31. DOI: 10.1590/ 2318-08892016002800002

33. Carvalho SBR, Araújo GC. Gestão da internacionalização das instituições de ensino superior. Avaliação (Campinas). 2020;25(1):113-31. DOI: $10.1590 /$ s1414-40772020000100007

34. Marques F. Os limites do índice-h. Pesquisa FAPESP [Internet]. 2013 [cited 2020 May 20];207:35-9. Available from: http://revistapesquisa.fapesp. br/2013/05/14/os-limites-do-indice-h/ 
35. Guimarães GL. Por uma crítica do índice-H pela área da Enfermagem à luz de Thomas Kuhn. Texto contexto enferm. 2019;28:e20180264. DOI: 10.1590/1980-265x-tce-2018-0264
Recebido: 2 de junho de 2020

Aprovado: 7 de julho de 2020

Publicado: 12 de agosto de 2020

A Revista Baiana de Enfermagem utiliza a Licença Creative Commons - Atribuição-NãoComercial 4.0 Internacional. https://creativecommons.org/licenses/by-nc/4.0/ Este artigo é de acesso aberto distribuído sob os termos da Licença Creative Commons (CC BY-NC). Esta licença permite que outros remixem, adaptem e criem a partir do seu trabalho para fins não comerciais. Embora os novos trabalhos tenham de lhe atribuir o devido crédito e não possam ser usados para fins comerciais, os usuários não têm de licenciar esses trabalhos derivados sob os mesmos termos. 